\title{
PREPRINTS NA COMUNICAÇÃO CIENTÍFICA: PROPOSTA DE MODELO PARA ACELERAÇÃO DO SISTEMA DE COMUNICAÇÃO CIENTÍFICA
}

\section{PREPRINTS IN SCIENTIFIC COMMUNICATION: MODEL PROPOSAL FOR ACCELERATING THE SCIENTIFIC COMMUNICATION SYSTEM}

Hemerson Soares da Silvaa

Fabiana Aparecida Lazzarin ${ }^{b}$

\begin{abstract}
RESUMO
Introdução: trate-se da criação de um modelo de aceleração do fluxo de comunicação científica empregando os repositórios digitais preprints. Objetivo: elaborar um modelo como meio para otimização do sistema de comunicação científica, empregando os seguintes objetivos específicos: a) refletir sobre aos aspectos teórico-conceituais a respeito dos meios de comunicação utilizado no sistema de comunicação científica; e, b) investigar possíveis caminhos alternativos para a aceleração do fluxo de comunicação científica. Metodologia: traça-se o percurso metodológico fundamentando-se no pensamento funcionalista, utilizando-se da pesquisa bibliográfica para refletir e compreender o papel dos preprints na comunicação científica por meio de uma análise de cunho qualitativo. Resultados: é apresentado o modelo de aceleração do sistema de comunicação fazendo uso dos repositórios digitais preprints. Conclusões: O modelo criado direcionará à aceleração do sistema de comunicação científica por meio dos repositórios digitais preprints e impulsionará e promoverá a colaboração entre os membros da comunidade científica.
\end{abstract}

Descritores: Comunicação científica. Repositórios digitais preprints. Modelo de aceleração.

\section{INTRODUÇÃO}

Desde o princípio, a sociedade buscou diversas maneiras de conhecer o mundo e explicar os fenômenos ao seu redor, e para isso, passou a utilizar métodos para validar as informações observadas, é partir daí que se tem o

\footnotetext{
a Mestrando no Programa de Pós-Graduação em Biblioteconomia na Universidade Federal do Cariri (PPGB/UFCA). E-mail: hemersonhsn@hotmail.com

b Professora do Curso de Biblioteconomia da Universidade Federal do Cariri (UFCA). E-mail: fabiana.lazzarin@ufca.edu.br
} 
surgimento da ciência. Logo, as pessoas passaram a observar, anotar, analisar e descrever suas percepções, compartilhando-as com os outros, assim, constituindo-se de forma preliminar o sistema de comunicação científica.

Segundo Meadows (1999), o ser humano é instigado a obter conhecimento, devido ao sentido que ele representa a si e as formas de sua aplicação. Na comunicação científica, as interações do ser humano com o mundo por meio de observações e experiências empíricas e a troca de ideias entre pesquisadores passaram a fazer parte do processo de comunicação científica.

Somando-se às ideias de Meadows (1999), segundo Weitzel (2006), os estudos acerca do fluxo do sistema de comunicação científica compreendem os aspectos inerentes específicos, sendo eles: geração, disseminação e uso do conhecimento científico.

Diante disso, Santos Junior (2010) frisa que o registro e divulgação dos resultados de pesquisa são fundamentais para geração de novos conhecimentos, e nesse contexto, os periódicos científicos se destacam como o principal canal de divulgação e validação da pesquisa. Apesar disso, recentemente com a introdução das tecnologias e a necessidade cada vez maior de divulgação rápida dos resultados de pesquisa em virtude da crescente demanda da comunidade científica, os repositórios digitais preprints têm demonstrado expressiva potencialidade na rápida divulgação das pesquisas.

Tal potencialidade já havia sido reconhecida nos estudos de Garvey e Griffith (1972) a respeito dos meios de comunicação de resultados no século XX, onde concluíram que os trabalhos em fase de pré-publicação (preprints) foram os meios mais utilizados para troca de ideias, e consequentemente, melhoria desses trabalhos. Dessa forma, percebendo suas vantagens, algumas comunidades científicas começaram a aderir aos repositórios preprints na tentativa de contornar o atraso das publicações em periódicos; no entanto, urge a necessidade da criação de um modelo.

Com base em tais pressupostos, formulou-se a seguinte questão: quais meios podem ser utilizados para aceleração no fluxo do sistema de comunicação científica? Perante à problemática, este estudo visa contribuir para às áreas do 
conhecimento, à comunidade científica, aos processos inerentes à divulgação de descobertas e aperfeiçoamento dos canais utilizados nas interações entre os membros da comunidade científica.

Em vista disso, tem-se como objetivo geral elaborar um modelo como meio para otimização do sistema de comunicação científica. Para isso, serão empregados os seguintes objetivos específicos: a) refletir sobre aos aspectos teórico-conceituais a respeito dos meios de comunicação utilizado no sistema de comunicação científica; e, b) investigar possíveis caminhos alternativos para a aceleração do fluxo da comunicação científica.

\section{PROCEDIMENTOS METODOLÓGICOS}

A pesquisa científica deve ser compreendida como a perscrutação da realidade de um mundo dinâmico, amplo e participativo preparada para lidar com diversas facetas a fim de encontrar respostas e soluções para às questões elencadas (LAZZARIN, 2014). Diante disso, traça-se o caminho metodológico fundamentando-se no funcionalismo. Utiliza-se métodos que analisam a função que os repositórios digitais preprints exercem sob o sistema de comunicação científica, e por conseguinte para com a sociedade, bem como as relações dos pesquisadores na troca de ideias e no uso dos canais de divulgação científica.

Para o delineamento da investigação, utilizou-se da pesquisa bibliográfica em materiais já consolidados na literatura científica, como artigos, livros, teses, dissertações, blogs, dicionários, anais de eventos, etc. (GIL, 2008).

Além disso, este estudo baseia-se na análise qualitativa, que segundo Gil (2008, p. 175) trata-se de uma técnica que não é composta por fórmulas ou receitas predefinidas, mas de uma análise que depende bastante da capacidade e do estilo do pesquisador. Dessa maneira, a pesquisa qualitativa revela áreas de consenso, tanto positivo quanto negativo, dentro das possíveis respostas, e é fundamental em cenários que envolvem o desenvolvimento e aperfeiçoamento de novas ideias (MORESI, 2003), como no contexto desta pesquisa, que é a busca por alternativas para otimização do fluxo da comunicação científica. 


\section{PERIÓDICO CIENTÍFICO: PERPASSANDO O PROCESSO DA COMUNICAÇÃO CIENTÍFICA EM DIREÇÃO AOS PREPRINTS}

Precedentemente aos primeiros mecanismos que moldaram o sistema de comunicação científica, a ciência era formada somente por filósofos que se utilizavam da argumentação e da dedução para explicar os fenômenos da natureza. Contudo, a partir do século XVII surge a ciência moderna, que por sua vez foi fomentada pela observação e experiência empírica, tais fatos culminaram no nascimento do periódico científico' (MUELLER, 2000).

Segundo Targino (1998), o periódico científico foi aceito formalmente como canal de comunicação entre os pesquisadores, e desde então as publicações em periódicos rapidamente expandiram-se em todo o mundo. Dialogando com Price (1976) o periódico científico, principalmente o periódico eletrônico trouxe diversas elucidações no que diz respeito a rápida divulgação de trabalhos atualizados frente ao crescente volume de informações na literatura que dificultava para que os pesquisadores se manterem atualizados. Nesse contexto, Mueller (2000, p. 81) reflete que o periódico eletrônico surge:

[...] como a esperança da solução há muito buscada, já que oferece mais rapidez na comunicação e flexibilidade de acesso, tem largo alcance e baixo custo relativo, disponibilidade imediata, é capaz de diminuir a necessidade de manutenção de coleções, barateando os custos.

Quanto a sua abrangência, o periódico eletrônico refere-se a todos os periódicos acessados através de equipamentos eletrônicos, e normalmente disponibilizados em mídias como CD-ROM e/ou via online. E no que concerne ao seu formato, os periódicos eletrônicos em sua maioria ainda são semelhantes aos periódicos impressos, já que também são disponibilizados em fascículos, possuem numeração e periodicidade; contudo, apresentam recursos distintos como hipertexto, inserção de sons, imagens e vídeos (MUELLER, 2000).

Além disso, é pertinente denotar algumas transformações proporcionadas pelos periódicos para a comunicação científica apresentadas no Quadro 1:

\footnotetext{
1 De acordo com Mueller (2000b) e Guédon (2001), o Philosophical Transactions foi o primeiro periódico científico, fundado em Londres no início de 1665.
} 


\section{Quadro 1 - Aspectos trazidos com a introdução do periódico eletrônico}

\begin{tabular}{|l|l|}
\hline Abrangência & \multicolumn{1}{|c|}{ Mudanças } \\
\hline $\begin{array}{l}\text { Bibliotecas e } \\
\text { bibliotecários }\end{array}$ & $\begin{array}{l}\text { os mecanismos de recuperação ficaram mais fáceis de utilizar, e os bibliotecários } \\
\text { especializados em pesquisa de banco de dados tornaram-se indispensáveis } \\
\text { (GUÉDON, 2001; HURD, 2004) }\end{array}$ \\
\hline Pesquisadores & $\begin{array}{l}\text { os pesquisadores passaram a utilizar o computador como instrumento de } \\
\text { pesquisa. Com o advento dos computadores de mesa na década de 1980, os } \\
\text { cientistas passaram a desenvolver funções que antes eram restritas ao setor } \\
\text { editorial (HURD, 2004) }\end{array}$ \\
\hline Autores & $\begin{array}{l}\text { trouxe poucas mudanças, já que ainda tinham que lidar com os periódicos, os } \\
\text { editores e a revisão por pares. Ressalta-se que umas das possíveis causas } \\
\text { esteja na falta de exploração dos recursos digitais uma vez que os artigos no } \\
\text { meio eletrônico são semelhantes ao impresso (GUÉDON, 2001) }\end{array}$ \\
\hline Leitores & $\begin{array}{l}\text { Em contrapartida, como leitores os pesquisadores rapidamente perceberam os } \\
\text { benefícios oferecidos como: copiar e transmitir documentos rapidamente, baixo } \\
\text { custo; e facilidade na recuperação de documentos (GUÉDON, 2001) }\end{array}$ \\
\hline $\begin{array}{l}\text { Formato do } \\
\text { artigo }\end{array}$ & $\begin{array}{l}\text { Introdução dos editores de texto eletrônico no artigo eletrônico, assim como os } \\
\text { recursos CrossRef, Digital Object Identifier (DOI), RefWorks }{ }^{2} \text { Open URL } \\
\text { mudaram os antigos papéis lineares para um ambiente mais complexo e inter- } \\
\text { relacionado na comunicação científica (HURD, 2004) }\end{array}$ \\
\hline
\end{tabular}

Fonte: elaborado pelos autores (2019).

Apesar dos diversos benefícios e soluções viabilizadas pelos periódicos eletrônicos, de acordo com Guédon (2001) a dificuldade de acesso aos artigos publicados nos periódicos ainda permanecia. Uma das causas que perpetua até hoje, trata-se da alta valorização do periódico científico como registro público ao invés de um meio de divulgação do conhecimento.

Tal crítica do referido autor, está relacionada com as práticas das editoras comerciais em cobrar altas taxas para aquisição de assinaturas de seus periódicos, dificultando para as bibliotecas que não dispunham de recursos suficientes pudessem adquirir todos os periódicos. Somado a isso, Guédon (2001) também aponta a dificuldade de digitalização dos documentos.

De acordo com Baptista et al. (2007) tais fatores provocaram a baixa visibilidade das pesquisas, assim prejudicando a disseminação e o acesso ao conhecimento científico. Por isso, diante desse cenário, gerou-se discussões a respeito da redução dos custos das assinaturas, bem como da busca por

2 Disponível em: https://www.exlibrisgroup.com/products/refworks-reference-management. Acesso em: 21 ago. 2018. 
alternativas de publicação.

Uma das primeiras alternativas mais conhecidas na literatura foi a criação do Science Citation Index (SCl), fundado pelo norte americano Eugene Garfield, diretor do Instituto de Informação Científica (IIC) (KURAMOTO, 2007). Segundo Guédon (2001; 2010), esse sistema funcionava como um núcleo de monitoramento de índice de citações dos periódicos, disponibilizando informações como periódicos mais relevantes e autores mais citados. O SCl facilitou para o crescimento dos periódicos e formação de coleções de qualidade nas bibliotecas, pois é uma ferramenta que auxilia os bibliotecários na seleção de materiais mais relevantes às necessidades dos usuários.

Contudo, é pertinente salientar que ao passo que o $\mathrm{SCl}$ favoreceu a ampla divulgação e estabeleceu mecanismos para monitoramento das produções científicas, como efeito negativo, Guédon (2001) aponta a intensificação do domínio das editoras comerciais.

Descontente com esse quadro, a comunidade científica implementou a Scholarly Publishing and Academic Resources Coalition (SPARC) com o intuito de oferecer periódicos eletrônicos com valores de assinatura mais baixo, e, por conseguinte, estimular as editoras comerciais a reduzirem os preços das assinaturas; no entanto, essa estratégia trouxe pouco impacto.

Posteriormente, emerge a chamada Open Access Initiative (OAI), também denominada por Movimento de Acesso Aberto. Dialogando com Baptista et al. (2007, p. 2), a finalidade da OAl abrange "[...] a disseminação ampla e irrestrita dos resultados de pesquisas financiadas com recursos", e estar alicerçada em manifestações como a Budapest Open Access Initiative (BOAl) originada pela Declaração de Budapest (2002), Declaração de Berlin (2003), Declaração de Bethesda (2003) e a Alhambra Declaration (2010).

Nesse sentido, Kuramoto (2006) afirma que esses movimentos têm como princípio de que todos os resultados de pesquisas financiadas com recursos públicos devem ser de acesso aberto à sociedade. Além disso, dialogando com Guédon (2001), Hurd (2004) e Baptista et al. (2007) a OAl adveio para vincular as coleções digitais às bibliotecas, e assim, contemplar características como: a) interoperabilidade dos dados; b) gerenciamento de direitos digitais; c) 
autoarquivamento das pesquisas em servidores de armazenamento, de modo a viabilizar a recuperação fácil e eficiente destas pesquisas; e, d) maior visibilidade das pesquisas e consequentemente, maximização de sua utilização pelos interessados.

Dentre os benefícios proporcionados pela OAI, Weitzel e Ferreira (2010) destaca a consolidação do modelo estrutural de bancos de dados na integração e no amplo acesso à produção científica na Internet, enquanto Santos Junior (2010) cita a ampliação da interação entre pesquisadores de diferentes instituições, facilidade no gerenciamento de todo conhecimento de uma instituição ou área em um só lugar e obtenção de dados sobre tudo que está sendo produzido.

Além disso, como a OAI tem uma filosofia de acesso gratuito, os autores dos trabalhos publicados não precisam se preocupar com questões de acesso indevido. Para reforçar a garantia de autoria, Gomes (2012) menciona a adoção de tecnologias de proteção como as licenças Creative Commons (CC) para que os pesquisadores possam criar, recriar, compartilhar, usar, reusar e disseminar legalmente a produção intelectual em prol da construção do conhecimento.

Em adição, Baptista et al. (2007) abordam duas estratégias para promoção do acesso aberto ao conhecimento científico: a via dourada e a via verde. No que concerne a via dourada, os célebres autores versam a publicação de artigos em periódicos de acesso aberto, isto é, periódicos que não possuem restrição de acesso e uso do material publicado, assim como a ausência de taxas para assinatura (normalmente elas utilizam outros métodos como taxas de publicação e publicidade para se manterem).

No caso da via verde, Santos Junior (2010) afirma que sua finalidade é disponibilizar os materiais de pesquisa publicamente na Web sem restrição de acesso, e nessa conjuntura, os repositórios são o principal canal para arquivamento e disponibilização desses materiais. Vale ressaltar que a implementação de repositórios digitais de acesso aberto requer uma boa infraestrutura tecnológica e a utilização de softwares como DSpace ${ }^{3}$.

\footnotetext{
${ }^{3}$ Disponível em: https://duraspace.org/dspace/about/. Acesso em: 5 ago. 2018.
} 
Em face ao explanado, percebe-se que as tecnologias da informação e comunicação e os movimentos a favor do acesso aberto influenciaram na forma de produzir, divulgar e utilizar o conhecimento no sistema de comunicação científica; assim, contribuindo para aceleração da produção científica, expansão do conhecimento que era ostensivamente restrito, e viabilização da criação de mecanismos para o acesso sem barreiras.

Em razão disso, o periódico eletrônico demonstrou ser um canal profusamente eficiente. Contudo, recentemente os repositórios digitais preprints têm se destacado como outra alternativa para a otimização do fluxo da comunicação científica, conforme será abordado mais adiante.

\section{PREPRINTS NA COMUNICAÇÃO CIENTÍFICA: ÊNFASE NA ACELERAÇÃO}

A produção, disseminação e uso da informação são elementos basilares para o fortalecimento das comunidades científicas. Desde a consolidação do sistema de comunicação científica, o ato de comunicar os resultados de pesquisa mediante dos canais formais e informais tornou-se indispensável para a construção do conhecimento, sobretudo, para aperfeiçoamento da ciência (ALVAREZ; CAREGNATO, 2017).

Nesse cenário, o periódico científico destacou-se como o canal mais bem aceito na publicação de trabalhos científicos. Pois, conforme Alvarez e Caregnato (2018) o processo de avaliação pelos pares nos periódicos constituise como importante mecanismo para a comunicação no campo científico, visto que é uma prática já amadurecida, apresentando eficiência na divulgação e certificação das pesquisas.

Não obstante, o periódico não é o único caminho para a comunicação científica, em virtude da rápida popularização dos repositórios preprints, trazendo várias inovações, dentre elas a aceleração de comunicação de descobertas entre os membros da comunidade científica, assim, preenchendo o espaço de tempo criado pelo atraso das publicações em periódicos.

No que diz respeito aos trabalhos preprints, Mueller (2000, p. 87) define preprint como "[...] nome dado à versão original de um artigo ainda não publicado 
oficialmente". Além disso, Søndergaard, Andersen e Hjørland (2003) acrescentam que os preprints são documentos distribuídos antes da sua publicação final em forma de artigo científico e antecipadamente à finalização da avaliação pelos pares.

Ainda sobre o conceito de preprints, para ASAPbio (2018) eles contêm dados e metodologias de pesquisa completos, permite atualizar o mesmo trabalho diversas vezes e normalmente não são avaliados pelos pares, como ocorre nos periódicos. Além disso, as versões preprints permitem que os pesquisadores aperfeiçoem seus trabalhos através do feedback da comunidade científica.

No que diz respeito às suas origens, uns dos precursores na descoberta dos preprints são Garvey e Griffith (1972), pois, ao estudar os meios utilizados no sistema de comunicação científica ainda em meados do século $X X$, os autores concluíram que, dentre os meios de divulgação e publicação de pesquisas, os preprints foram os mais utilizados para divulgação prévia dos resultados da pesquisa. Nesse universo, os pesquisadores faziam uso do preprint com o intuito de aperfeiçoar sua pesquisa, uma vez que o compartilhamento prévio de descobertas com seus pares revelava-se como oportunidade para aprimoramento de suas ideias.

Para os autores supracitados, o uso de preprints beneficia tanto o autor quanto os seus leitores. Nesse seguimento, o leitor tem o privilégio de obter informações relevantes durante o andamento da pesquisa ao passo que os autores:

[...] recebem feedback que os leva a modificar seus manuscritos. Essas modificações não são simplesmente uma questão de melhoria na gramática e estilo do manuscrito, mas, em vez disso, envolvem modificações significativas, como reanálise de dados, redefinição de conceitos, etc. Consequentemente, a distribuição de preprints é, para muitos autores, um meio efetivo de obter opiniões independentemente do valor científico de seu trabalho (GARVEY; GRIFFITH, 1972, p. 131, tradução dos autores).

Garvey e Griffith (1972) também afirmam que o uso dos preprints agiliza o processo de publicação do artigo científico, uma vez que o manuscrito é submetido ao periódico, sua versão preprint pode ser acessada enquanto aguarda-se a publicação da versão final, isto é, enquanto a submissão ainda 
passará por avaliação e ajustes, os leitores já podem ter acesso prévio ao seu conteúdo através da pré-publicação.

Tendo em consideração os benefícios dos preprints, surge em agosto de 1991, o primeiro repositório digital de preprints, denominado por $\mathrm{ArXiv}^{4}$, que por sua vez foi fundado por Paul Ginsparg para uso na área de conhecimento, a física (GINSPARG, 1997).

Segundo Ginsparg (1997), o ArXiv passou a oferecer recursos automatizados, como por exemplo, processo de submissão e indexação de dados do documento, acesso via e-mail, Internet, File Transfer Protoco/5 (FTP), permitindo que os pesquisadores se comunicassem por meio de conversas em grupos virtuais. Percebendo as vantagens proporcionadas pelo ArXiv, gradualmente emergem diversas iniciativas de repositórios digitais preprints em todo mundo, incluindo áreas como Matemática, Ciências da Computação, Biologia, Finanças e Estatística (JACOBSEN, 2017).

Em continuidade, Moreno, Leite e Arellano (2006) descrevem os repositórios como meio de acesso público transparente, suporte a uma ampla tipologia de documentos, conteúdo heterogêneo, multidisciplinaridade e preservação digital do conhecimento científico.

Apesar dos inúmeros benefícios, os preprints não possuem em sua essência a avaliação pelos pares como nos artigos científicos, por isso, Guimarães, Silva e Noronha (2009) assentam que os repositórios não vieram para competir e/ou substituir os periódicos científicos, mas para complementar e potencializar o sistema de comunicação científica. Costa e Leite (2010) corroboram ao afirmarem que os repositórios potencializariam o registro, arquivamento e acesso ao conhecimento científico, enquanto os periódicos continuariam com o papel de validação e qualificação dos resultados de pesquisa.

No que se refere ao seu funcionamento, em síntese os repositórios digitais preprints funcionam da seguinte maneira: os trabalhos são depositados; em

\footnotetext{
${ }^{4}$ Disponível em: https://arxiv.org/. Acesso em: 20 ago. 2019.

${ }^{5}$ Em português "Protocolo de Transferência de Arquivos", muito utilizado para transferência de arquivos entre computadores na rede.
} 
seguida são liberados para acesso público após passar por um filtro; essa filtragem é conduzida por um moderador, que fica responsável por avaliar se o trabalho está de acordo com os critérios estabelecidos pelo repositório; se aceito, o trabalho é divulgado imediatamente (BAPTISTA et al., 2007).

No que toca às questões inerentes ao plágio, é interessante destacar a facilidade em identificar trabalhos plagiados, visto que há ampla disponibilidade "[...] ao mesmo tempo, aumentam exponencialmente o registro da autoria pois, quanto mais disponível e acessível um trabalho, mais sua propriedade intelectual é reforçada" (COSTA; LEITE, 2010, p. 170). Outra vantagem assinalada por Correia (2001), diz respeito que todo trabalho publicado em repositórios preprints pode ser citado em periódicos científico, já que gradualmente os periódicos estão aceitando artigos científicos que citam trabalhos preprints.

Além disso, a visibilidade proporcionada pelos preprints de acordo com a ASAPbio (2018) pode ser um convite para reuniões onde os interessados procuram trabalhos mais recentes e relevantes ainda não publicados em periódicos, o uso do feedback por meio dos comentários de outros pesquisadores permite que $\mathrm{o}$ trabalho seja aperfeiçoado e a prioridade de descobertas e ideias.

Ademais, é importante mencionar as mudanças que os repositórios digitais preprints proporcionam às bibliotecas, nesse sentido, Costa e Leite (2010, p. 181) aludem que "[...] novos e relevantes papéis passam a ser desempenhados por seus bibliotecários, além de ser aumentada significativamente a parceria destes com pesquisadores no exercício de suas funções".

Em vista disso, as bibliotecas de universidades e de institutos de pesquisa desempenham novos papéis no que concerne à editoração científica, isto é, as bibliotecas tornam-se um dos principais atores no processo de comunicação científica, especialmente na publicação científica, e ainda:

[...] bibliotecários e editores podem ser colocados lado a lado, no sentido em que realizam tarefas semelhantes de divulgação e distribuição dos resultados do trabalho de pesquisadores. Funcionam, desse modo, como mediadores do processo de comunicação entre emissores e receptores do conhecimento resultado de pesquisas (COSTA; LEITE, 2010, p. 183). 
Trazendo para o contexto dos repositórios preprints, o bibliotecário atua como mediador entre o pesquisador e a informação científica, dado que sua atuação é imprescindível para promoção do acesso ao conhecimento pela comunidade científica.

Devido a potencialidade dos preprints, Hurd (2000) aponta que a adoção de uma cultura de uso dos repositórios preprints valoriza a rápida difusão dos resultados preliminares, acelera a comunicação entre pesquisadores, viabiliza a atuação ativa dos colégios invisíveis, fortalece a cooperação e elimina as barreiras geográficas entre diferentes grupos de pesquisa, conforme ilustrado na Figura 1:

Figura 1 - Papel dos repositórios preprints na comunicação científica

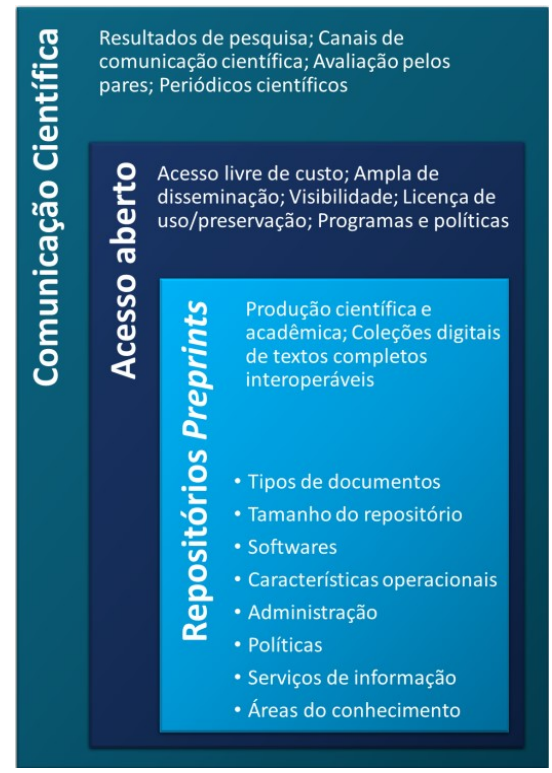

Fonte: Adaptado de Moreno, Leite e Arellano (2006), Costa e Leite (2017).

Nesse contexto, Alvarez e Caregnato (2017) ponderam que os repositórios digitais preprints nascem de necessidades da própria comunidade científica em produzir e disseminar pesquisas consoante a atual realidade da comunicação científica, na qual a divulgação rápida e precisa dos resultados é fundamental para o desenvolvimento da ciência. 


\section{PROPOSTA DE MODELO PARA ACELERAÇÃO DO SISTEMA DE COMUNICAÇÃO CIENTÍFICA}

Quando se observa a trajetória do sistema de comunicação científica, constata-se múltiplas transformações em seu fluxo, sobretudo no comportamento da comunidade científica frente aos instrumentos e canais de comunicação de pesquisa. No contexto global, as tecnologias ampliaram as possibilidades de comunicação que tempos atrás não era imaginado.

No cenário evidenciado, especialmente com o advento da Internet, emergem novas ferramentas e estratégias revolucionárias para o compartilhamento de ideias, como os repositórios preprints. Por isso, diante dessa discussão teórico-conceitual, foi proposto o seguinte modelo para aceleração da comunicação científica através dos repositórios preprints, conforme apresentado na Figura 2. 


\section{Figura 2 - Papel dos repositórios preprints na comunicação científica}

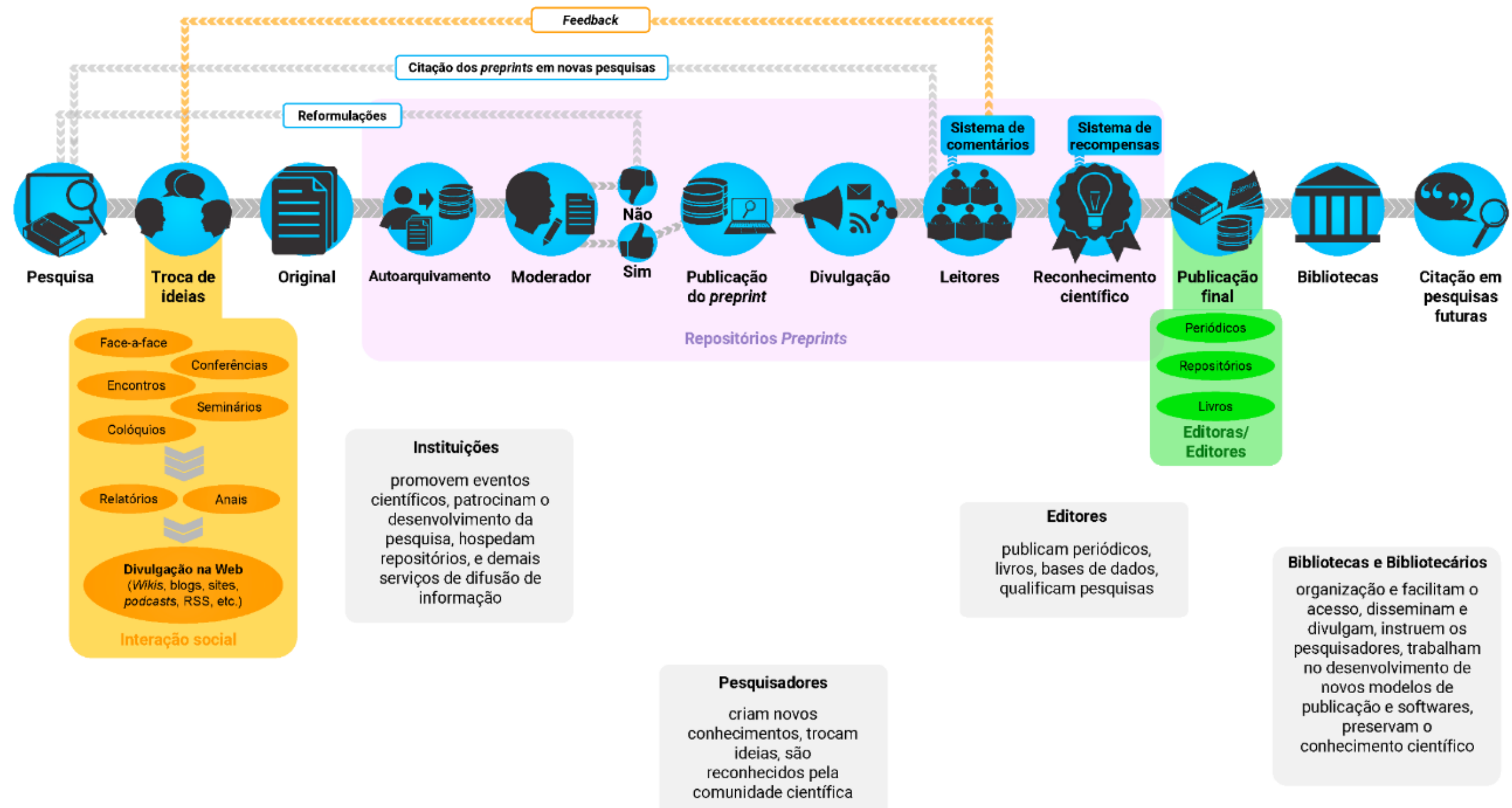

Fonte: Elaborado pelos autores (2019). 
Pesquisa: na fase inicial o pesquisador faz uso de instrumentos, consulta a literatura já consolidada na ciência (bibliotecas, livros, periódicos, repositórios, etc.), e ainda delineia os objetivos, hipóteses e metodologia da pesquisa.

Troca de ideias: durante a pesquisa, a troca de ideias se destaca como importante meio para divulgação e obtenção de conhecimento, nesse processo o pesquisador poderá fazer uso de eventos, produção de relatórios, anais e aplicações Web para trocar ideias. Além disso, os pesquisadores já podem utilizar os repositórios preprints para troca de ideias com autores de outros trabalhos publicados nesse canal.

Original: na elaboração dos originais, além dos autores descrevem os procedimentos e suas observações, é possível utilizar recursos como o hipertexto e as referências linkadas a fim de facilitar aos leitores a localização dos documentos citados.

Autoarquivamento: na submissão, o repositório deve ter uma interface intuitiva de fácil manuseio, e ainda permitir a inserção dos dados como título, autor, data, resumo, palavras-chave e anexar outros arquivos ao manuscrito.

Moderador: nesta etapa, o moderador tem o papel de analisar se a pesquisa atende os critérios pré-estabelecidos pelo repositório. Normalmente, essa avaliação é rápida e o trabalho fica disponível em até um dia.

Publicação do preprint: uma vez aprovado, o preprint fica disponível abertamente à comunidade científica.

Divulgação: a divulgação abrange a indexação em bases de dados, bibliotecas, motores de busca e sites com finalidade de divulgação de conteúdo científico. Uma das vantagens dos repositórios é que os pesquisadores podem optar em receber notificações somente dos assuntos de seu interesse.

Leitores: rapidamente os leitores têm acesso às publicações, nos repositórios é possível baixar os documentos em qualquer computador e a qualquer momento, bem como podem dar o feedback sobre suas impressões para os autores da pesquisa. Como o feedback também é um processo de troca de ideias, os autores podem aperfeiçoar a pesquisa e submeter novamente uma versão atualizada do preprint no repositório, assim permitindo a construção de trabalhos com melhor qualidade científica. 
Reconhecimento científico: através do reconhecimento o pesquisador percebe o valor do seu esforço no desenvolvimento de seus estudos, além de ser uma forma de incentivar à produção de novas pesquisas.

Publicação final: a publicação definitiva é realizada em outros canais de divulgação, como os periódicos (que continuará com a avaliação pelos pares), livros e outros repositórios. Além disso, é importante frisar que as editoras continuarão exercendo papel especial como difusoras de trabalhos científicos de qualidade nos canais formais da comunicação científica.

Bibliotecas: continuarão exercendo sua função como difusora do conhecimento científico, e os bibliotecários poderão desenvolver coleções com os trabalhos científicos mais reconhecidos pela comunidade científica e conforme o perfil dos usuários. Ademais, os bibliotecários através dos serviços de referência poderão auxiliar os pesquisadores na busca de informação, na utilização das ferramentas de busca, repositórios, periódicos, etc., bem como demais tecnologias da biblioteca.

Citação em pesquisas futuras: por fim, os trabalhos divulgados em diversos tipos de canais são citados em pesquisas futuras. É nessa etapa que se impulsiona a geração de novos conhecimentos em futuros estudos.

Em síntese, este modelo vai além da aceleração da divulgação de novas descobertas, como também impulsiona e promove a colaboração dos pares, assim contribuindo para concepção de pesquisas de qualidade.

\section{CONSIDERAÇÕES FINAIS}

Em face às reflexões concernentes às transformações no fluxo da comunicação científica, depreende-se que essas expressivas mudanças alcançaram em as diversas nuances e atores envolvidos. Diante da problemática levantada neste estudo, compreende-se que uma das alternativas para otimização do fluxo do sistema de comunicação científica é a proposta de um novo modelo introduzindo os repositórios digitais preprints em seu fluxo.

Entende-se que há uma diversidade de meios utilizados para a comunicação de resultados de pesquisa que contemplaram as necessidades dos 
pesquisadores em diferentes contextos no desenvolvimento da ciência. Além disso, percebe-se que esses meios evoluíram expressivamente no decorrer do tempo, e portanto, influenciando o fluxo da informação científica e o comportamento dos pesquisadores.

Dessa forma, foi instigado investigar caminhos alternativos para a aceleração do sistema de comunicação científica, que nesse cenário, os repositórios digitais preprints destacaram-se como meio de difusão eficiente da informação científica, contemplando tanto os pesquisadores quanto às áreas, disciplinas, instituições de pesquisa, editoras, bibliotecas e bibliotecários.

Portanto, foi possível criar um modelo direcionado à aceleração do sistema de comunicação científica por meio dos repositórios digitais preprints. Além disso, tal modelo vai além da aceleração ao impulsionar e promover a colaboração entre os membros da comunidade científica.

\section{REFERÊNCIAS}

ALHAMBRA DECLARATION ON OPEN ACCESS. 2010. Disponível em: https://www.jlis.it/article/view/8632/7775. Acesso em: 5 ago. 2018.

ALVAREZ, G. R.; CAREGNATO, S. E. Open pre-review: avaliação de preprints em repositórios. SciELO 20 Years Repository, p. 1-7, set. 2018. Disponível em: http://repository.scielo20.org/documents/article/view/103. Acesso em: 2 jan. 2019.

ALVAREZ, G. R.; CAREGNATO, S. E. Preprints na comunicação científica da Física de Altas Energias: análise das submissões no repositório arXiv (20102015). Perspectivas em Ciência da Informação, v. 22, n. 2, p. 104-117, abr./jun. 2017. Disponível em:

http://portaldeperiodicos.eci.ufmg.br/index.php/pci/article/view/2830. Acesso em: 17 dez. 2018.

ASAPBIO. Preprint FAQ. ASAPbio: São Franscisco, CA, 2018. Disponível em: http://asapbio.org/preprint-info/preprint-faq. Acesso em: 4 abr. 2018.

BAPTISTA, A.; COSTA, S.; KURAMOTO, H.; RODRIGUES, E. Comunicação científica: o papel da open archives initiative no contexto do acesso livre.

Encontros Bibli: revista eletrônica de biblioteconomia e ciência da informação, Florianópolis, p. 1-17, dez. 2007. Disponível em: https://periodicos.ufsc.br/index.php/eb/article/view/15182924.2007v12nesp1p1/435. Acesso em: 10 mar. 2018. 
BERLIN DECLARATION ON OPEN ACCESS TO KNOWLEDGE IN THE SCIENCES AND HUMANITIES. [S. I.: s. n.], 2003. Disponível em: https://openaccess.mpg.de/2365/en. Acesso em: 11 mar. 2018.

BETHESDA STATEMENT ON OPEN ACCESS PUBLISHING. Released June 20, 2003. [S. I.: s. n.], 2003. Disponível em:

http://www.earlham.edu/ peters/fos/bethesda.htm. Acesso em: 10 mar. 2018.

BUDAPEST OPEN ACCESS INITIATIVE (BOAI). Read the Budapest Open Access Initiative. [S. I.: s. n.], 2002. Disponível em: http://www.budapestopenaccessinitiative.org/read. Acesso em: 5 ago. 2018.

CORREIA, A. M. R. O papel das bibliotecas digitais de literatura científica cinzenta: os repositórios de e-prints na comunicação científica. In: JBIDI. 2001. Disponível em: http://infonautica.net/docs/jbidi/jbidi2001/23_2001.pdf. Acesso em: 17 dez. 2018.

COSTA, S. M. S.; LEITE, F. C. L. Insumos conceituais e práticos para iniciativas de repositórios institucionais de acesso aberto à informação científica em bibliotecas de pesquisa. In: SAYÃO, L. F. et al. Implantação e gestão de repositórios institucionais: políticas, memória, livre acesso e preservação. Salvador: EDUFBA, 2010. p. 163-202.

GARVEY, W. D.; GRIFFITH, B. C. Communication and information processing within scientific disciplines: empirical findings for psychology. Information storage and retrieval, v. 8, p. 123-136, 1972. Disponível: https://ac.elscdn.com/0020027172900411/1-s2.0-0020027172900411main.pdf? tid=de722a52-0b6a-11e8-9b3e00000aacb̄35d\&acdnat=1517941606_fa6d581a404a715d054a4a9c7e21e905. Acesso em: 6 fev. 2018.

GIL, A. C. Métodos e técnicas de pesquisa social. 6. ed. São Paulo: Atlas, 2008. 200 p.

GINSPARG, P. Winners and losers in the global research village. The Serials Librarian, v. 3-4, n. 30, p. 83-95, 1997. Disponível em: https://www.tandfonline. com/doi/pdf/10.1300/J123v30n03_13. Acesso em: 17 dez. 2018.

GOMES, C. M. Comunicação científica: cartografia e desdobramentos. 325 p. 2012. Tese (Doutorado), Programa de Pós-graduação em Ciência da Informação - Escola de Comunicação e Artes, Universidade de São Paulo, 2012.

GUÉDON, J. Acesso aberto e divisão entre ciência predominante e ciência periférica. In: FERREIRA, S. M. S. P; TARGINO, M. G. (org.). Acessibilidade e visibilidade de revistas científicas. São Paulo: Senac; Cengage Learning, 2010. p. 21-78. 
GUÉDON, J. In Oldenburg's long shadow: librarians, research scientists, publishers, and the control of scientific publishing. Association of Research Libraries, Washington DC, 2001. Disponível em: http://www.arl.org/storage/ documents/publications/in-oldenburgs-long-shadow.pdf. Acesso em: 11 jan. 2018

GUIMARÃES, M. C. S.; SILVA, C. H.; NORONHA, I. M. H. RI é a resposta, mas qual é a pergunta? Primeiras anotações para a implementação de repositório institucional. In: SAYÃO, L. F. et al. (org.). Implantação e gestão de repositórios institucionais: políticas, memória, livre acesso e preservação. Salvador: EDUFBA, 2009. p. 261-281.

HURD, J. M. Scientific Communication: new roles and new players. The Haworth Information Press, v. 25, n. 1/2, p. 5-22, 2004. Disponível em: http://jacklandunited.com/JacklandUnited/LBSC601_Readings_files/Hurd.pdf. Acesso em: 17 dez. 2018.

HURD, J. M. The transformation of scientific communication: a model for 2020. Journal of the American Society for Information Science, v. 51, p. 12791283, 2000. Disponível em: https://pdfs.semanticscholar.org/51c0/3c5ce775 7ac89f22cb7559f85caded54c75c.pdf. Acesso em: 6 fev. 2018.

JACOBSEN, P. Preprints: o caminho a seguir para o compartilhamento rápido e aberto do conhecimento. Blog da BC, 2017. Disponível em:

https://www.ufrgs.br/blogdabc/preprints-o-caminho-a-seguir-para-ocompartilhamento-rapido-e-aberto-do-conhecimento/. Acesso em: 4 abr. 2018.

KURAMOTO, H. Acesso livre: um caso de soberania nacional? In: TOUTAIN, L. M. B. B. (org.). Para entender a Ciência da Informação. Salvador: EDUFBA, 2007. p. 145-161.

KURAMOTO, H. Informação científica: proposta de um novo modelo para o Brasil. Ci. Inf., Brasília, v. 35, n. 2, p. 91-102, maio/ago. 2006. Disponível em: http://www.scielo.br/pdf/ci/v35n2/a10v35n2.pdf. Acesso em: 14 ago. 2018.

LAZZARIN, F. A. De olho no OPAC da biblioteca universitária: avaliação sobre e-acessibilidade e arquitetura da informação para Web com a interação de usuários cegos. 2014. 224 f. Dissertação (Mestrado) - Programa de PósGraduação em Ciência da Informação, Universidade Federal da Paraíba, João Pessoa, 2014.

MEADOWS, A. J. Mudança e crescimento. In: MEADOWS, A. J. A comunicação científica. Brasília: Briquet de Lemos/Livros, 1999. p. 1-38.

MORENO, F. P.; LEITE, F. C. L.; ARELLANO, M. Á. M. Acesso livre a publicações e repositórios digitais em Ciência da Informação no Brasil. Perspectivas em Ciência da Informação, v. 11, n. 1, p. 255-269, 2006. Disponível em: 
http://www.repositorio.unb.br/bitstream/10482/623/1/ARTIGO_AcessoLivrePubli cacoes.pdf. Acesso em: 10 mar. 2018.

MORESI, E. (org.). Metodologia da pesquisa. Brasília, DF: Universidade Católica de Brasília, Pró-Reitoria de Pós-graduação - PRPG, 2003. 108 p.

MUELLER, S. P. M. O periódico científico. In: CAMPELLO, B. S.; CENDÓN, B. V.; KREMER, J. M. (org.). Fontes de informação para pesquisadores e profissionais. Belo Horizonte: UFMG, 2000. p. 72-95.

PRICE, F. J. S. O desenvolvimento da ciência. Rio de Janeiro: Livros Técnicos e Científicos, 1976. 77 p.

SANTOS JUNIOR, E. R. Repositórios institucionais de acesso livre no Brasil: estudo Delfos. 2010. 177 f. Dissertação (Mestrado em Ciência da Informação) - Universidade de Brasília, Brasília, 2010.

SØNDERGAARD, T. F.; ANDERSEN, J.; HJØRLAND, B. Documents and the communication of scientific and scholarly information: revising and updating the UNISIST model. Journal of Documentation, v. 59, n. 3, p. 278-320, 2003. Disponível em: https://pdfs.semanticscholar.org/0f1d/8159d2bb33fb222 79c5ee5799ac424e806b1.pdf. Acesso em: 17 dez. 2018.

TARGINO, M. G. Comunicação científica. In: TARGINO, M. G. Comunicação científica: o artigo de periódico nas atividades de ensino e pesquisa do docente universitário brasileiro na pós-graduação. 1998. p. 31-88. Tese (Doutorado em Ciência da Informação) - Departamento de Ciência da Informação e Documentação da Faculdade de Estudos Sociais Aplicados da Universidade de Brasília, Brasília: UnB, 1998.

WEITZEL, S. R. Os repositórios de e-prints como nova forma de organização da produção científica: o caso da área das Ciências da Comunicação no Brasil. 2006. 361 f. Tese (Doutorado em Ciência da Informação) - Universidade de São Paulo, São Paulo, 2006.

WEITZEL, S. R; FERREIRA, S. M. S. P. Percepção sobre acesso e visibilidade dos repositórios digitais e das revistas eletrônicas. In: FERREIRA, S. S. P.; TARGINO, M. G. (org.). Acessibilidade e visibilidade de revistas científicas. São Paulo: Senac; São Paulo: Cengage Learning, 2010. p. 119-172.

\title{
PREPRINTS IN SCIENTIFIC COMMUNICATION: MODEL PROPOSAL FOR ACCELERATING THE SCIENTIFIC COMMUNICATION SYSTEM
}

\author{
ABSTRACT \\ Introduction: This is a study about the creation of a scientific communication flow
}


acceleration model using digital preprint repositories. Objective: To develop a model as a means of optimizing the scientific communication system, employing the following specific objectives: a) to reflect on the theoretical and conceptual aspects regarding the media used in the scientific communication system; and, b) investigate possible alternative ways to accelerate the flow of scientific communication. Methodology: The methodological course is traced based on functionalist thinking, using bibliographic research to reflect and understand the role of preprints in scientific communication through a qualitative analysis. Results: The acceleration model of the communication system using the preprint digital repositories is presented. Conclusions: The created model will direct the acceleration of the scientific communication system through the preprint digital repositories, going beyond acceleration by boosting and promoting collaboration among the members of the scientific community.

Descriptors: Scientific communication. Digital preprints repositories. Acceleration model.

\title{
PREPRINTS EN COMUNICACIÓN CIENTÍFICA: PROPUESTA MODELO PARA ACELERAR EL SISTEMA DE COMUNICACIÓN CIENTÍFICA
}

\begin{abstract}
RESUMEN
Introducción: Este es un estudio sobre la creación de un modelo de aceleración del flujo de comunicación científica utilizando repositorios de preimpresión digital. Objetivo: Desarrollar un modelo como medio para optimizar el sistema de comunicación científica, empleando los siguientes objetivos específicos: a) reflexionar sobre los aspectos teóricos y conceptuales con respecto a los medios utilizados en el sistema de comunicación científica; y, b) investigar posibles formas alternativas de acelerar el flujo de la comunicación científica. Metodología: el curso metodológico se basa en el pensamiento funcionalista, utilizando la investigación bibliográfica para reflejar y comprender el papel de las preimpresiones en la comunicación científica a través de un análisis cualitativo. Resultados: se presenta el modelo de aceleración del sistema de comunicación que utiliza los repositorios digitales de preimpresión. Conclusiones: El modelo creado dirigirá la aceleración del sistema de comunicación científica a través de los repositorios digitales de preimpresión, yendo más allá de la aceleración al impulsar y promover la colaboración entre los miembros de la comunidad científica.
\end{abstract}

Descriptores: Comunicación científica. Repositorios de preimpresiones digitales. Modelo de aceleración.

Recebido em: 05.09.2019

Aceito em: 09.09.2019 